\title{
Quasi-stability and continuity of attractors for nonlinear system of wave equations
}

https://doi.org/10.1515/msds-2020-0125

Received November 22, 2020; accepted March 8, 2021

Abstract: In this paper, we study the long-time behavior of a nonlinear coupled system of wave equations with damping terms and subjected to small perturbations of autonomous external forces. Using the recent approach by Chueshov and Lasiecka in [21], we prove that this dynamical system is quasi-stable by establishing a quasistability estimate, as consequence, the existence of global and exponential attractors is proved. Finally, we investigate the upper and lower semicontinuity of global attractors under autonomous perturbations.

Keywords: Wave equations; quasi-stable systems; global attractor; exponential attractor; continuity of attractors

MSC: 35B40; 35B41; 35L05; 35L75

\section{Introduction}

In this paper, we are interested in the long-time behavior of the coupled system of wave equations given by

$$
\begin{cases}u_{t t}-\Delta u+(-\Delta)^{\alpha_{1}} u_{t}+g_{1}\left(u_{t}\right)=f_{1}(u, v)+\epsilon h_{1}, & \text { in } \Omega \times \mathbb{R}^{+}, \\ v_{t t}-\Delta v+(-\Delta)^{\alpha_{2}} v_{t}+g_{2}\left(v_{t}\right)=f_{2}(u, v)+\epsilon h_{2}, & \text { in } \Omega \times \mathbb{R}^{+}, \\ u=v=0, & \text { on } \partial \Omega \times \mathbb{R}^{+}, \\ u(0)=u_{0}, u_{t}(0)=u_{1}, & \text { in } \Omega, \\ v(0)=v_{0}, v_{t}(0)=v_{1}, & \text { in } \Omega .\end{cases}
$$

Here $\Omega \subset \mathbb{R}^{2}$ is a bounded domain with a smooth boundary $\partial \Omega, \alpha_{1}, \alpha_{2} \in(0,1)$ and $\epsilon \in[0,1]$. The nonlinearities $f_{1}(u, v), f_{2}(u, v)$ are with supercritical exponents representing strong sources, $(-\Delta)^{\alpha_{1}} u_{t}$ and $(-\Delta)^{\alpha_{2}} v_{t}$ are the fractional damping, while $g_{1}\left(u_{t}\right)$ and $g_{2}\left(v_{t}\right)$ act as nonlinear damping. The terms $\epsilon h_{1}, \epsilon h_{2}$ are autonomous perturbations of external forces.

In the literature there is a large number of work with damping and source terms see, e.g., $[1,2,24,32,39$ 42] for problems with subcritical or critical sources, and [9-12, 22, 23, 26-28, 43] for problems with supercritical sources and with fractional damping see, e.g., [3, 7, 13-19, 34, 38, 46] and references therein.

\footnotetext{
^Corresponding Author: M. M. Freitas: Faculty of Mathematics, Federal University of Pará, Raimundo Santana Cruz Street, S/N, 68721-000, Salinópolis, Pará, Brazil, E-mail: mirelson@ufpa.br

M. J. Dos Santos: Faculty of Exact Sciences and Technology, Federal University of Pará, Manoel de Abreu St, Abaetetuba, PA, 68440-000, Brazil, E-mail: jeremias@ufpa.br

A. J. A. Ramos: Faculty of Mathematics, Federal University of Pará, Raimundo Santana Cruz Street, S/N, 68721-000, Salinópolis, Pará, Brazil, E-mail: ramos@ufpa.br

M. S. Vinhote: PhD Program in Mathematics, Federal University of Pará, Augusto Corrêa Street, 01, 66075-110, Belém, Pará, Brazil, E-mail: mvinhote25@gmail.com

M. L. Santos: PhD Program in Mathematics, Federal University of Pará, Augusto Corrêa Street, 01, 66075-110, Belém, Pará, Brazil, E-mail: 1s@ufpa.br
} 
In [28], Guo et al. studied the local and global well-posedness of the coupled nonlinear wave equations

$$
\begin{cases}u_{t t}-\Delta u+g_{1}\left(u_{t}\right)=f_{1}(u, v), & \text { in } \Omega \times(0, T), \\ v_{t t}-\Delta v+g_{2}\left(v_{t}\right)=f_{2}(u, v), & \text { in } \Omega \times(0, T),\end{cases}
$$

in a bounded domain $\Omega \subset \mathbb{R}^{n}$ with a nonlinear Robin boundary condition on $u$ and a zero boundary conditions on $v$. By employing nonlinear semigroups and the theory of monotone operators, the authors obtained several results on the existence of local and global weak solutions, and uniqueness of weak solutions. Moreover, they proved that such unique solutions depend continuously on the initial data. Under some restrictions on the parameters, they also proved that every weak solution to our system blows up in finite time, provided the initial energy is negative and the sources are more dominant than the damping in the system.

The present system (1.1) is obtained by the model (1.2) by adding the fractional dissipations $(-\Delta)^{\alpha_{1}} u_{t},(-\Delta)^{\alpha_{2}} v_{t}$ and considering small perturbations of autonomous external forces $\epsilon h_{1}, \epsilon h_{2}$. Our main interest in this paper is to study the long-time behavior of the autonomous dynamical system generated by the nonlinear coupled system of wave equations (1.1). In this context, the concept of global attractor is a useful objective to learn the dynamical behavior of a dynamical system [5, 8, 21, 29, 33, 44, 45]. By using the quasi-stability theory of Chueshov and Lasiecka [20, 21], we prove that the dynamical system generated by the problem (1.1) possesses a compact global attractor with finite fractal dimension. We also prove the regularity of solutions on the global attractor. Moreover, we obtain the existence of a generalized exponential attractor with fractal dimension finite in extended spaces. We also established the stability of global attractors on the perturbation of the parameter $\epsilon$. More precisely, we use the recent theory in [31] to prove that there exists a set $I_{\star}$ dense in $[0,1]$ such that the family of global attractors $\left\{\mathcal{A}_{\epsilon}\right\}_{\epsilon \in[0,1]}$ associated to problem (1.1) converges upper and lower-semicontinuously to the corresponding global attractor associated with the limit problem when the parameter $\epsilon \rightarrow \epsilon_{0}$ for all $\epsilon_{0} \in I_{*}$. Moreover, the upper semicontinuity for all $\epsilon \in[0,1]$ is analyzed.

The main contributions of this paper are:

(i) We consider the system with fractional and nonlinear dissipation acting on the same equation. Here, we assume the nonlinear damping terms with polynomial growth to include functions of type $g_{i}(u)=|u|^{p-1} u$.

(ii) Instead of showing the existence of an absorbing set, we prove the system is gradient, and hence obtain the existence of a global attractor, which is characterized as unstable manifold of the set of stationary solutions.

(iii) The quasi-stability of the system is obtained by establishing a quasistability estimate and therefore obtain the finite dimensionality and smoothness of the global attractor and exponential attractor.

(iv) We investigate the continuity of the family of global attractors under autonomous perturbations. Indeed, we prove that the family of global attractors indexed by $\epsilon$ converges upper and lower-semicontinuously to the attractor associated with the limiting problem when $\epsilon \rightarrow \epsilon_{0}$ on a residual dense set $I_{\star} \subset[0,1]$ in the same sense proposed in Hoang et al. [31]. The upper semicontinuity or all $\epsilon \in[0,1]$ is proved.

This paper is organized as follows. In section 2, we introduce some notations and preliminary results. We also give the well-posedness of the system without proof in this section. Section 3 is devoted to proving the existence of global attractors and exponential attractors. The arguments are based on the methods developed by Chueshov and Lasiecka [20, 21]. The continuity of global attractors will be proved in section 4 .

\section{Preliminaries}

In this section, we present preliminaries including notations and assumptions. 


\subsection{Notations and assumptions}

The following notations will be used for the rest of the paper.

$$
\|u\|_{p}=\|u\|_{L^{p}(\Omega)}, \quad p \geq 1, \quad(u, v)=(u, v)_{L^{2}(\Omega)} .
$$

In this work, we consider the Hilbert space

$$
\mathcal{H}=H_{0}^{1}(\Omega) \times H_{0}^{1}(\Omega) \times L^{2}(\Omega) \times L^{2}(\Omega),
$$

with the following inner product and norm

$$
(U, \tilde{U})_{\mathcal{H}}=(\phi, \tilde{\phi})+(\varphi, \tilde{\varphi})+(\nabla u, \nabla \tilde{u})+(\nabla v, \nabla \tilde{v})
$$

and

$$
\|U\|_{\mathcal{H}}^{2}=\|\phi\|_{2}^{2}+\|\varphi\|_{2}^{2}+\|\nabla u\|_{2}^{2}+\|\nabla v\|_{2}^{2}
$$

for any $U=(u, v, \phi, \varphi)$ and $\tilde{U}=(\tilde{u}, \tilde{v}, \tilde{\phi}, \tilde{\varphi})$ in $\mathcal{H}$.

Assumption 2.1. We assume that

(i) The external forces $h_{1}, h_{2} \in L^{2}(\Omega)$.

(ii) There is a function $F \in C^{2}\left(\mathbb{R}^{2}\right)$ such that

$$
\nabla F=\left(f_{1}, f_{2}\right)
$$

and there exist $p \geq 1$ and $C>0$ such that

$$
\left|\nabla f_{i}(u, v)\right| \leq C\left(1+|u|^{p-1}+|v|^{p-1}\right), \quad \forall u, v \in \mathbb{R} .
$$

(iii) There is $\beta_{0}>0$ and $m_{F}>0$ so that

$$
F(u, v) \leq \beta_{0}\left(|u|^{2}+|v|^{2}\right)+m_{F}, \quad \forall u, v \in \mathbb{R},
$$

where $0 \leq \beta_{0}<\frac{\lambda_{1}}{2}$ and $\lambda_{1}>0$ denotes the Poincaré's constant. Moreover

$$
\nabla F(u, v) \cdot(u, v)-F(u, v) \leq \beta_{0}\left(|u|^{2}+|v|^{2}\right)+m_{F}, \quad \forall u, v \in \mathbb{R} .
$$

(iv) Concerning to the nonlinear damping $g_{i}$, we assume that

$$
g_{i} \in C^{1}(\mathbb{R}), g_{i}(0)=0,
$$

and there exists constants $m, M_{1}, q \geq 1$, such that

$$
m \leq g_{i}^{\prime}(u) \leq M_{1}\left(1+|u|^{q-1}\right), \quad \forall u \in \mathbb{R},
$$

and, if $q \geq 3$, there exist $l>q-1$ and $M_{2}>0$ such that

$$
g_{i}(u) u \geq M_{2}|u|^{l}, \quad|u| \geq 1 .
$$

Remark 2.1. Observe that assumption (2.11) implies the monotonicity property, that is,

$$
\left(g_{i}(u)-g_{i}(v)\right)(u-v) \geq m|u-v|^{2}, \quad \forall u, v \in \mathbb{R} .
$$

Remark 2.2. An simple example of the function $F$ in Assumption 2.1 can be

$$
F(u, v)=-|u+v|^{4}+|u+v|^{2}-c_{1}|u v|^{2}, \quad c_{1}>0 .
$$

In this case, we have

$$
\begin{aligned}
& f_{1}(u, v)=\frac{\partial F}{\partial u}=-4(u+v)^{3}+2(u+v)-2 c_{1} u v^{2} \\
& f_{2}(u, v)=\frac{\partial F}{\partial v}=-4(u+v)^{3}+2(u+v)-2 c_{1} u^{2} v
\end{aligned}
$$


Since

$$
F(u, v) \leq \max _{\xi \in \mathbb{R}}\left\{-\xi^{4}+\xi^{2}\right\}=\frac{1}{4},
$$

the assumptions (2.7)-(2.8) hold with $m_{F}=\frac{1}{4}$ and $p=3$. Noting that

$$
\nabla F(u, v) \cdot(u, v)-F(u, v) \leq-3|u+v|^{4}+|u+v|^{2} \leq \frac{1}{12} \leq m_{F},
$$

then (2.9) also holds.

\subsection{Energy identities}

The total energy of system (1.1) is defined by as

$$
E(t)=\frac{1}{2}\left\|\left(u, v, u_{t}, v_{t}\right)\right\|_{\mathcal{H}}^{2}=\frac{1}{2}\left(\left\|u_{t}\right\|_{2}^{2}+\left\|v_{t}\right\|_{2}^{2}+\|\nabla u\|_{2}^{2}+\|\nabla v\|_{2}^{2}\right),
$$

and we also define the total energy by

$$
\varepsilon_{\epsilon}(t)=E(t)-\int_{\Omega} F(u, v) d x-\epsilon \int_{\Omega}\left(h_{1} u+h_{2} v\right) d x .
$$

Then we have the following lemma.

Lemma 2.1. The total energy given in (2.15) satisfies

$$
\frac{d}{d t} \varepsilon_{\epsilon}(t) \leq-\left(\left\|(-\Delta)^{\frac{\alpha_{1}}{2}} u_{t}\right\|_{2}^{2}+\left\|(-\Delta)^{\frac{\alpha_{2}}{2}} v_{t}\right\|_{2}^{2}\right)-m\left(\left\|u_{t}\right\|_{2}^{2}+\left\|v_{t}\right\|_{2}^{2}\right) .
$$

Moreover, there exists a positive constant $C_{0}$ such that

$$
C_{0}\left\|\left(u, v, u_{t}, v_{t}\right)\right\|_{\mathcal{H}}^{2}-C_{F} \leq \mathcal{E}_{\epsilon}(t) \leq C_{F}\left(1+\left\|\left(u, v, u_{t}, v_{t}\right)\right\|_{\mathcal{H}}^{p+1}\right), \quad \forall \epsilon \in[0,1] .
$$

Proof. Multiplying (formally) the first equation in (1.1) by $u_{t}$, and second by $v_{t}$, respectively and using integration by parts, we can easily get (2.16). It follows from (2.8) and Poincaré's inequality that

$$
\begin{aligned}
\int_{\Omega} F(u, v) d x & \leq \beta_{0}\left(\|u\|_{2}^{2}+\|v\|_{2}^{2}\right)+m_{F}|\Omega| \\
& \leq \frac{\beta_{0}}{\lambda_{1}}\left(\|\nabla u\|_{2}^{2}+\|\nabla v\|_{2}^{2}\right)+m_{F}|\Omega| \\
& \leq \frac{\beta_{0}}{\lambda_{1}}\left\|\left(u, v, u_{t}, v_{t}\right)\right\|_{\mathcal{H}}^{2}+m_{F}|\Omega|,
\end{aligned}
$$

and thus

$$
\varepsilon_{\epsilon}(t) \geq\left(\frac{1}{2}-\frac{\beta_{0}}{\lambda_{1}}\right)\left\|\left(u, v, u_{t}, v_{t}\right)\right\|_{\mathcal{H}}^{2}-m_{F}|\Omega|-\epsilon \int_{\Omega}\left(h_{1} u+h_{2} v\right) d x
$$

Letting

$$
C_{0}=\frac{1}{4}\left(1-\frac{2 \beta_{0}}{\lambda_{1}}\right)>0
$$

and using the estimate

$$
\epsilon \int_{\Omega}\left(h_{1} u+h_{2} v\right) d x \leq C_{0} \lambda_{1}\left(\|u\|_{2}^{2}+\|v\|_{2}^{2}\right)+\frac{1}{4 C_{0} \lambda_{1}}\left(\left\|h_{1}\right\|_{2}^{2}+\left\|h_{2}\right\|_{2}^{2}\right),
$$

we obtain the first inequality in (2.17) with

$$
C_{F}=m_{F}|\Omega|+\frac{1}{4 C_{0} \lambda_{1}}\left(\left\|h_{1}\right\|_{2}^{2}+\left\|h_{2}\right\|_{2}^{2}\right) .
$$

Using (2.7) we can see that the second inequality in (2.17) holds. The proof is complete. 


\subsection{Well-posedness}

Let us write the problem (1.1) as an equivalent Cauchy problem

$$
\left\{\begin{array}{l}
\frac{d U}{d t}+\mathbb{A} U=\mathbb{F}(U), \\
U(0)=U_{0}=\left(u_{0}, v_{0}, u_{1}, v_{1}\right) \in \mathcal{H},
\end{array}\right.
$$

where

$$
U(t)=(u(t), v(t), \phi(t), \varphi(t)) \in \mathcal{H}, \quad \phi=u_{t}, \quad \varphi=v_{t},
$$

and $\mathcal{H}$ defined in (2.3) and $\mathbb{A}: D(\mathbb{A}) \subset \mathcal{H} \rightarrow \mathcal{H}$ is the nonlinear operator defined by

$$
\mathbb{A} U=\left(\begin{array}{c}
-\phi \\
-\varphi \\
-\Delta u+(-\Delta)^{\alpha_{1}} \phi+g_{1}(\phi) \\
-\Delta v+(-\Delta)^{\alpha_{2}} \varphi+g_{2}(\varphi)
\end{array}\right) .
$$

The domain of $\mathbb{A}$ is given by

$$
\begin{aligned}
D(\mathbb{A})=\{ & U=(u, v, \phi, \varphi) \in \mathcal{H}: \phi \in H_{0}^{1}(\Omega), \varphi \in H_{0}^{1}(\Omega), \\
& -\Delta u+(-\Delta)^{\alpha_{1}} \phi+g_{1}(\phi) \in L^{2}(\Omega), \\
& \left.-\Delta v+(-\Delta)^{\alpha_{2}} \varphi+g_{2}(\varphi) \in L^{2}(\Omega)\right\} .
\end{aligned}
$$

The forcing terms are represented by a nonlinear function $\mathbb{F}: \mathcal{H} \rightarrow \mathcal{H}$ defined by

$$
\mathbb{F}(U)=\left(\begin{array}{c}
0 \\
0 \\
f_{1}(u, v)+\epsilon h_{1} \\
f_{2}(u, v)+\epsilon h_{2}
\end{array}\right) .
$$

We have the following global existence result for the problem (1.1).

Theorem 2.2. Suppose that Assumption 2.1 holds, then we have:

(i) If initial data $U_{0} \in \mathcal{H}$, then problem $(2.20)$ has a unique mild solution $U(t) \in C([0, \infty)$, $\mathcal{H})$ with $U(0)=U_{0}$ given by

$$
U(t)=e^{\mathbb{A} t} U_{0}+\int_{0}^{t} e^{(t-\tau) \mathbb{A}} \mathbb{F}(U(\tau)) d \tau .
$$

(ii) If $U_{0} \in D(\mathbb{A})$, then the above mild solution is a strong solution.

(iii) If $U^{1}(t)$ and $U^{2}(t)$ are two mild solutions of problem (2.20) then there exists a positive constant $C_{0}=$ $C\left(U^{1}(0), U^{2}(0)\right)$, such that

$$
\left\|U^{1}(t)-U^{2}(t)\right\|_{\mathcal{H}} \leq e^{C_{0} T}\left\|U^{1}(0)-U^{2}(0)\right\|_{\mathcal{H}}, \quad 0 \leq t \leq T .
$$

Proof. It is easy to see that the operator $\mathbb{A}$ is a maximal monotone operator. In addition, by (2.7) we see that $\mathbb{F}$ is a locally Lipschitz on $\mathcal{H}$. Therefore, applying the theory of maximal nonlinear monotone operators (see e.g. $[6,21]$ ) items (i)-(ii) are concluded. The continuous dependence (iii) is also obtained by using standard computations in the difference of solutions.

\section{Long-time dynamics}

On account of Theorem 2.2, we can define the one-parameter family of operators $S_{\epsilon}(t): \mathcal{H} \rightarrow \mathcal{H}$ by

$$
S_{\epsilon}(t)\left(u_{0}, v_{0}, u_{1}, v_{1}\right)=\left(u(t), v(t), u_{t}(t), v_{t}(t)\right), \quad t \geq 0,
$$


where $\left(u, v, u_{t}, v_{t}\right)$ is the unique solution of problem (1.1). Thus, the pair $\left(\mathcal{H}, S_{\epsilon}(t)\right)$ constitutes a dynamical system that will describe the long-time behavior of problem (1.1).

The main result for long-time dynamics is given in the following theorem.

Theorem 3.1. Under the assumptions of Theorem 2.2, we have:

(i) The dynamical system $\left(\mathcal{H}, S_{\epsilon}(t)\right)$ given in (3.23) is quasi-stable on any bounded positively invariant set $B \subset$ $\mathcal{H}$.

(ii) The dynamical system $\left(\mathcal{H}, S_{\epsilon}(t)\right)$ possesses a unique compact global attractor $\mathcal{A}_{\epsilon} \subset \mathcal{H}$, which is characterized by the unstable manifold $\mathcal{A}_{\epsilon}=\mathbb{M}_{+}\left(\mathcal{N}_{\epsilon}\right)$ of the set of stationary solutions

$$
\mathcal{N}_{\epsilon}=\left\{\begin{array}{l|l}
(u, v, 0,0) \in \mathcal{H} & \begin{array}{l}
-\Delta u=f_{1}(u, v)+\epsilon h_{1} \\
-\Delta u=f_{2}(u, v)+\epsilon h_{2}
\end{array}
\end{array}\right\} .
$$

(iii) Every trajectory stabilizes to the set $\mathcal{N}_{\epsilon}$, namely, for any $U \in \mathcal{H}$ one has

$$
\lim _{t \rightarrow+\infty} \operatorname{dist}_{\mathcal{H}}\left(S(t) U, \mathcal{N}_{\epsilon}\right)=0 .
$$

In particular, there exists a global minimal attractor $\mathcal{A}_{\epsilon}^{\min }$ given by $\mathcal{A}_{\epsilon}^{\min }=\mathcal{N}_{\epsilon}$.

(iv) The attractor $\mathcal{A}_{\epsilon}$ has finite fractal and Hausdorff dimension $\operatorname{dim}_{\mathcal{H}}^{f} \mathcal{A}_{\epsilon}$.

(v) If $\alpha_{i} \in(0,1 / 2), i=1,2$, the global attractor $\mathcal{A}_{\epsilon}$ is bounded in

$$
\mathcal{H}_{1}=\left(H^{2}(\Omega) \cap H_{0}^{1}(\Omega)\right)^{2} \times\left(H_{0}^{1}(\Omega)\right)^{2} .
$$

Moreover, every trajectory $U=\left(u, v, u_{t}, v_{t}\right)$ in $\mathcal{A}_{\epsilon}$ satisfies

$$
\|(u, v)\|_{\left(H^{2}(\Omega) \cap H_{0}^{1}(\Omega)\right)^{2}}^{2}+\left\|\left(u_{t}, v_{t}\right)\right\|_{\left(H_{0}^{1}(\Omega)\right)^{2}}^{2}+\left\|\left(u_{t t}, v_{t t}\right)\right\|_{\left(L^{2}(\Omega)\right)^{2}}^{2} \leq R_{1}^{2},
$$

for some constant $R_{1}>0$ independent of $\epsilon \in[0,1]$.

(vi) The dynamical system $\left(\mathcal{H}, S_{\epsilon}(t)\right)$ possesses a generalized fractal exponential attractor. More precisely, for any $\delta \in(0,1]$, there exists a generalized exponential attractor $\mathcal{A}_{\epsilon, \delta}^{\exp } \subset \mathcal{H}$, with finite fractal dimension in extended space $\widetilde{\mathcal{H}}_{-\delta}$, defined as interpolation of

$$
\widetilde{\mathcal{H}}_{0}:=\mathcal{H} \text {, and } \widetilde{\mathcal{H}}_{-1}:=\left[L^{2}(\Omega) \times H^{-1}(\Omega)\right]^{2} .
$$

The proof of this theorem will be achieved in the end of this section.

\subsection{Quasistability estimate}

The aim of this section is to derive quasistability estimate which is the main tool in proving finite dimensionality and smoothness of attractors.

Lemma 3.2. Suppose that Assumptions 2.1 holds. Let $B \subset \mathcal{H}$ be a positively invariant bounded subset and let $S_{\epsilon}(t) U^{i}=\left(u^{i}(t), v^{i}(t), u_{t}^{i}(t), v_{t}^{i}(t)\right), \quad i=1,2$, be weak solution of (1.1) with initial conditions $U^{i} \in B$. Then there exist constants $\omega_{B}, \vartheta_{B}, C_{B}>0$ independent of $\epsilon$ such that

$$
E(t) \leq \vartheta_{B} E(0) e^{-\omega_{B} t}+C_{B} \sup _{\sigma \in[0, t]}\left(\|u(\sigma)\|_{2 \theta}^{2}+\|v(\sigma)\|_{2 \theta}^{2}\right), \quad \forall t \geq 0,
$$

for some $\theta \geq 2$, where $u=u^{1}-u^{2}$ and $v=v^{1}-v^{2}$.

Proof. Using the notations

$$
F_{i}(u, v)=f_{i}\left(u^{1}, v^{1}\right)-f_{i}\left(u^{2}, v^{2}\right), G_{1}(u)=g_{1}\left(u^{1}\right)-g_{1}\left(u^{2}\right), G_{2}(v)=g_{2}\left(v^{1}\right)-g_{2}\left(v^{2}\right),
$$


then, the differences $u=u^{1}-u^{2}$ and $v=v^{1}-v^{2}$ satisfies

$$
\begin{cases}u_{t t}-\Delta u+(-\Delta)^{\alpha_{1}} u_{t}+G_{1}\left(u_{t}\right)=F_{1}(u, v), & \text { in } \Omega \times(0, T), \\ v_{t t}-\Delta v+(-\Delta)^{\alpha_{2}} v_{t}+G_{2}\left(v_{t}\right)=F_{2}(u, v), & \text { in } \Omega \times(0, T),\end{cases}
$$

with boundary Dirichlet conditions and initial data

$$
\left(u(0), v(0), u_{t}(0), v_{t}(0)\right)=U^{1}-U^{2} .
$$

Multiplying the equations in (3.26) by $u$ and $v$, respectively, and integrating over $[0, T] \times \Omega$, yields

$$
\begin{aligned}
\int_{0}^{T} E(t) d t= & -\left.\frac{1}{2} \int_{\Omega}\left(u u_{t}+v v_{t}\right) d x\right|_{0} ^{T}+\int_{0}^{T}\left(\left\|u_{t}\right\|_{2}^{2}+\left\|v_{t}\right\|_{2}^{2}\right) d t \\
& -\frac{1}{2} \int_{0}^{T} \int_{\Omega}\left((-\Delta)^{\frac{\alpha_{1}}{2}} u_{t}(-\Delta)^{\frac{\alpha_{1}}{2}} u+(-\Delta)^{\frac{\alpha_{2}}{2}} v_{t}(-\Delta)^{\frac{\alpha_{2}}{2}} v\right) d x d t \\
& -\frac{1}{2} \int_{0}^{T} \int_{\Omega}\left(G_{1}\left(u_{t}\right) u+G_{2}\left(v_{t}\right) v\right) d x d t \\
& +\frac{1}{2} \int_{0}^{T} \int_{\Omega}\left(F_{1}(u, v) u+F_{2}(u, v) v\right) d x d t .
\end{aligned}
$$

We shall estimate the right-hand side of (3.28).

Step 1. From Hölder's and Poincarés inequalities, we deduce

$$
\left.\int_{\Omega}\left(u u_{t}+v v_{t}\right) d x\right|_{0} ^{T} \leq C(E(T)+E(0))
$$

for some constant $C>0$. Using the assumption (2.13), we obtain

$$
\int_{0}^{T}\left(\left\|u_{t}\right\|_{2}^{2}+\left\|v_{t}\right\|_{2}^{2}\right) d t \leq \frac{1}{m} \int_{0}^{T} \int_{\Omega}\left(G_{1}\left(u_{t}\right) u_{t}+G_{2}\left(v_{t}\right) v_{t}\right) d x d t .
$$

Step 2. By using the Young's inequality, continuous embedding $H_{0}^{1}(\Omega) \hookrightarrow D\left((-\Delta)^{\frac{s}{2}}\right)$ for $0<s<1$, we find

$$
\begin{aligned}
\int_{0}^{T} \int_{\Omega}(-\Delta)^{\frac{\alpha_{1}}{2}} u_{t}(-\Delta)^{\frac{\alpha_{1}}{2}} u d x d t & \leq \int_{0}^{T}\left\|(-\Delta)^{\frac{\alpha_{1}}{2}} u_{t}\right\|_{2}\left\|(-\Delta)^{\frac{\alpha_{1}}{2}} u\right\|_{2} d t \\
& \leq C \int_{0}^{T}\|\nabla u\|_{2}\left\|(-\Delta)^{\frac{\alpha_{1}}{2}} u_{t}\right\|_{2} d t \\
& \leq \frac{1}{4} \int_{0}^{T}\|\nabla u\|_{2}^{2} d t+C \int_{0}^{T}\left\|(-\Delta)^{\frac{\alpha_{1}}{2}} u_{t}\right\|_{2}^{2} d t .
\end{aligned}
$$

Analogously,

$$
\int_{0}^{T} \int_{\Omega}(-\Delta)^{\frac{\alpha_{2}}{2}} v_{t}(-\Delta)^{\frac{\alpha_{2}}{2}} v d x d t \leq \frac{1}{4} \int_{0}^{T}\|\nabla v\|_{2}^{2} d t+C \int_{0}^{T}\left\|(-\Delta)^{\frac{\alpha_{2}}{2}} v_{t}\right\|_{2}^{2} d t
$$

Then

$$
\begin{aligned}
& \int_{0}^{T} \int_{\Omega}\left((-\Delta)^{\frac{\alpha_{1}}{2}} u_{t}(-\Delta)^{\frac{\alpha_{1}}{2}} u+(-\Delta)^{\frac{\alpha_{2}}{2}} v_{t}(-\Delta)^{\frac{\alpha_{2}}{2}} v\right) d x d t \\
& \leq \frac{1}{2} \int_{0}^{T} E(t) d t+C \int_{0}^{T}\left(\left\|(-\Delta)^{\frac{\alpha_{1}}{2}} u_{t}\right\|_{2}^{2}+\left\|(-\Delta)^{\frac{\alpha_{2}}{2}} v_{t}\right\|_{2}^{2}\right) d t .
\end{aligned}
$$


Step 3. From Young's inequality and (2.13), we obtain

$$
\int_{0}^{T} \int_{\Omega} G_{1}\left(u_{t}\right) u d x d t \leq \frac{1}{2} \int_{0}^{T} \int_{\Omega} G_{1}\left(u_{t}\right) u_{t} d x d t+\frac{1}{2} \int_{0}^{T} \int_{\Omega} G_{1}\left(u_{t}\right) \frac{|u|^{2}}{u_{t}} d x d t .
$$

Using the assumption (2.11), we deduce

$$
\int_{\Omega} G_{1}\left(u_{t}\right) u d x \leq \frac{1}{2} \int_{\Omega} G_{1}\left(u_{t}\right) u_{t} d x+\frac{M_{1}}{2} \int_{\Omega}\left(1+\left|u_{t}^{1}\right|^{q-1}+\left|u_{t}^{2}\right|^{q-1}\right)|u|^{2} d x .
$$

To estimate the second term in the right side of (3.31), we consider three cases separately: Case 1. $q=1$. In this case, it is easy see that

$$
\int_{\Omega}\left(1+\left|u_{t}^{1}\right|^{q-1}+\left|u_{t}^{2}\right|^{q-1}\right)|u|^{2} d x \leq 3\|u\|_{2}^{2}\left(1+\int_{\Omega}\left(g_{1}\left(u_{t}^{1}\right) u_{t}^{1}+g_{1}\left(u_{t}^{2}\right) u_{t}^{2}\right) d x\right) .
$$

Case 2. $q \geq 3$. For this situation we use (2.12) and Holder's inequality to get

$$
\begin{aligned}
\int_{\Omega}\left(1+\left|u_{t}^{1}\right|^{q-1}+\left|u_{t}^{2}\right|^{q-1}\right)|u|^{2} d x & \leq C\|u\|_{\frac{2 l}{l-q+1}}^{2}\left(\int_{\Omega}\left(1+\left|u_{t}^{1}\right|^{l}+\left|u_{t}^{2}\right|^{l}\right) d x\right)^{\frac{q-1}{l}} \\
& \leq C\|u\|_{\frac{2 l}{l-q+1}}^{2}\left(\int_{\Omega}\left(1+g_{1}\left(u_{t}^{1}\right) u_{t}^{1}+g_{1}\left(u_{t}^{2}\right) u_{t}^{2}\right) d x\right)^{\frac{q-1}{l}} \\
& \leq C\|u\|_{\frac{2 l}{l-q+1}}^{2}\left(\int_{\Omega}\left(1+g_{1}\left(u_{t}^{1}\right) u_{t}^{1}+g_{1}\left(u_{t}^{2}\right) u_{t}^{2}\right) d x\right)
\end{aligned}
$$

where we have used the fact that $\frac{q-1}{l}<1$.

Case 3. $1<q<3$. As in case 2 we obtain

$$
\begin{aligned}
\int_{\Omega}\left(1+\left|u_{t}^{1}\right|^{q-1}+\left|u_{t}^{2}\right|^{q-1}\right)|u|^{2} d x & \leq C\|u\|_{\frac{4}{3-q}}^{2}\left(\int_{\Omega}\left(1+\left|u_{t}^{1}\right|^{2}+\left|u_{t}^{2}\right|^{2}\right) d x\right)^{\frac{q-1}{2}} \\
& \leq C\|u\|_{\frac{4}{3-q}}^{2}\left(\int_{\Omega}\left(1+g_{1}\left(u_{t}^{1}\right) u_{t}^{1}+g_{1}\left(u_{t}^{2}\right) u_{t}^{2}\right) d x\right)^{\frac{q-1}{2}} \\
& \leq C\|u\|_{\frac{4}{3-q}}^{2}\left(\int_{\Omega}\left(1+g_{1}\left(u_{t}^{1}\right) u_{t}^{1}+g_{1}\left(u_{t}^{2}\right) u_{t}^{2}\right) d x\right)
\end{aligned}
$$

Combining the three last estimates and (3.31) we conclude that there exist $C>0$ and $\theta \geq 2$ such that

$$
\int_{\Omega} G_{1}\left(u_{t}\right) u d x \leq \frac{1}{2} \int_{\Omega} G_{1}\left(u_{t}\right) u_{t} d x+C\|u\|_{\theta}^{2}\left(\int_{\Omega}\left(1+g_{1}\left(u_{t}^{1}\right) u_{t}^{1}+g_{1}\left(u_{t}^{2}\right) u_{t}^{2}\right) d x\right) .
$$

Analogously,

$$
\int_{\Omega} G_{2}\left(v_{t}\right) v d x \leq \frac{1}{2} \int_{\Omega} G_{2}\left(v_{t}\right) v_{t} d x+C\|v\|_{\theta}^{2}\left(\int_{\Omega}\left(1+g_{2}\left(v_{t}^{1}\right) v_{t}^{1}+g_{1}\left(v_{t}^{2}\right) v_{t}^{2}\right) d x\right) .
$$


Then, since $U^{1}, U^{2} \in B$, we deduce that by (2.16) and (2.17) that there exists $C_{B}>0$ such that

$$
\begin{aligned}
& \int_{\Omega}\left(1+g_{1}\left(u_{t}^{1}\right) u_{t}^{1}+g_{1}\left(u_{t}^{2}\right) u_{t}^{2}\right) d x \leq C_{B}, \\
& \int_{\Omega}\left(1+g_{2}\left(v_{t}^{1}\right) v_{t}^{1}+g_{1}\left(v_{t}^{2}\right) v_{t}^{2}\right) d x \leq C_{B} .
\end{aligned}
$$

Combining the last estimate with (3.32) and (2.12) and using the embedding $L^{2 \theta}(\Omega) \hookrightarrow L^{\theta}(\Omega)$ we obtain

$$
\begin{aligned}
-\frac{1}{2} \int_{0}^{T} \int_{\Omega}\left(G_{1}\left(u_{t}\right) u+G_{2}\left(v_{t}\right) v\right) d x d t \leq & \frac{1}{2} \int_{0}^{T} \int_{\Omega}\left(G_{1}\left(u_{t}\right) u_{t}+G_{2}\left(v_{t}\right) v_{t}\right) d x d t \\
& +C_{B, T} \sup _{\sigma \in[0, T]}\left(\|u(\sigma)\|_{2 \theta}^{2}+\|v(\sigma)\|_{2 \theta}^{2}\right) .
\end{aligned}
$$

Step 4. Using (2.7), the Hölder's inequality with conjugated exponents $\tilde{\theta}=\frac{2 \theta}{\theta-1}, 2 \theta$ and 2, and the embedding (in 2D) $H_{0}^{1}(\Omega) \hookrightarrow L^{S}(\Omega), 1 \leq s<\infty$, we obtain

$$
\begin{aligned}
\int_{\Omega} F_{1}(u, v) u d x & \leq C\left(\nabla f_{1}\right)\left(\|u\|_{2 \theta}+\|v\|_{2 \theta}\right)\|v\|_{2} \\
& \leq C_{B}\left(\|u\|_{2 \theta}+\|v\|_{2 \theta}\right)\|v\|_{2} \\
& \leq C_{B}\left(\|u\|_{2 \theta}^{2}+\|v\|_{2 \theta}^{2}\right)
\end{aligned}
$$

where

$$
C\left(\nabla f_{1}\right)=C\left(1+\left\|u^{1}\right\|_{(p-1) \tilde{\theta}}^{p-1}+\left\|u^{2}\right\|_{(p-1) \tilde{\theta}}^{p-1}+\left\|v^{1}\right\|_{(p-1) \tilde{\theta}}^{p-1}+\left\|v^{2}\right\|_{(p-1) \tilde{\theta}}^{p-1}\right) .
$$

Analogously,

$$
\int_{\Omega} F_{2}(u, v) v d x \leq C_{B}\left(\|u\|_{2 \theta}^{2}+\|v\|_{2 \theta}^{2}\right)
$$

Combining the two last estimates, there exists $C_{B, T}>0$ such that

$$
\frac{1}{2} \int_{0}^{T} \int_{\Omega}\left(F_{1}(u, v) u+F_{2}(u, v) v\right) d x d t \leq C_{B, T} \sup _{\sigma \in[0, T]}\left(\|u(\sigma)\|_{2 \theta}^{2}+\|v(\sigma)\|_{2 \theta}^{2}\right) .
$$

Inserting the estimates (3.29)-(3.35) into (3.28), we conclude that there exist $C_{B}>0$ and $C_{B, T}>0$ such that

$$
\begin{aligned}
\int_{0}^{T} E(t) d t \leq & C(E(T)+E(0)) \\
& +C_{B} \int_{0}^{T}\left(\left\|(-\Delta)^{\frac{\alpha_{1}}{2}} u_{t}\right\|_{2}^{2}+\left\|(-\Delta)^{\frac{\alpha_{2}}{2}} v_{t}\right\|_{2}^{2}\right) d t \\
& +C_{B} \int_{0}^{T} \int_{\Omega}\left(G_{1}\left(u_{t}\right) u_{t}+G_{2}\left(v_{t}\right) v_{t}\right) d x d t \\
& +C_{B, T} \sup _{\sigma \in[0, T]}\left(\|u(\sigma)\|_{2 \theta}^{2}+\|v(\sigma)\|_{2 \theta}^{2}\right) .
\end{aligned}
$$


Step 5. Multiplying the equation in (3.26) by $u_{t}$ and $v_{t}$, respectively, and then integrating over $[s, T] \times \Omega$, yields

$$
\begin{aligned}
E(T)= & E(s)-\int_{0}^{T}\left(\left\|(-\Delta)^{\frac{\alpha_{1}}{2}} u_{t}\right\|_{2}^{2}+\left\|(-\Delta)^{\frac{\alpha_{2}}{2}} v_{t}\right\|_{2}^{2}\right) d t \\
& -\int_{0}^{T} \int_{\Omega}\left(G_{1}\left(u_{t}\right) u_{t}+G_{2}\left(v_{t}\right) v_{t}\right) d x d t \\
& +\int_{0}^{T} \int_{\Omega}\left(F_{1}(u, v) u_{t}+F_{2}(u, v) v_{t}\right) d x d t .
\end{aligned}
$$

Since

$$
-\int_{0}^{T} \int_{\Omega}\left(G_{1}\left(u_{t}\right) u_{t}+G_{2}\left(v_{t}\right) v_{t}\right) d x d t \leq 0
$$

we have

$$
E(T) \leq E(s)+\int_{0}^{T} \int_{\Omega}\left(F_{1}(u, v) u_{t}+F_{2}(u, v) v_{t}\right) d x d t
$$

Similarly to (3.34), we deduce that

$$
\begin{aligned}
\int_{\Omega} F_{1}(u, v) u_{t} d x & \leq C_{B}\left(\|u\|_{2 \theta}+\|v\|_{2 \theta}\right)\left\|u_{t}\right\|_{2} \\
& \leq \epsilon\left\|u_{t}\right\|_{2}^{2}+\frac{C_{B}}{4 \epsilon}\left(\|u\|_{2 \theta}^{2}+\|v\|_{2 \theta}^{2}\right) .
\end{aligned}
$$

Similarly,

$$
\int_{\Omega} F_{2}(u, v) v_{t} d x \leq \epsilon\left\|v_{t}\right\|_{2}^{2}+\frac{C_{B}}{4 \epsilon}\left(\|u\|_{2 \theta}^{2}+\|v\|_{2 \theta}^{2}\right) .
$$

Therefore,

$$
\int_{\Omega}\left(F_{1}(u, v) u_{1}+F_{2}(u, v) v_{t}\right) d x \leq \epsilon E(t)+\frac{C_{B}}{4 \epsilon}\left(\|u\|_{2 \theta}^{2}+\|v\|_{2 \theta}^{2}\right) .
$$

Inserting the last estimate into (3.38) with $\epsilon=\frac{1}{T}$, we find that

$$
E(T) \leq E(s)+\frac{1}{T} \int_{0}^{T} E(t) d t+T C_{B} \int_{0}^{T}\left(\|u\|_{2 \theta}^{2}+\|v\|_{2 \theta}^{2}\right) d t
$$

Integrating the last estimate over $[0, T]$ with respect to $\mathrm{s}$, we conclude that there exist a constant $C_{B, T}>0$ such that

$$
T E(T) \leq 2 \int_{0}^{T} E(t) d t+C_{B, T} \sup _{\sigma \in[0, T]}\left(\|u(\sigma)\|_{2 \theta}^{2}+\|v(\sigma)\|_{2 \theta}^{2}\right) .
$$

Step 6. The estimates (3.37) and (3.41) with $\epsilon=1$ imply

$$
\begin{gathered}
\int_{0}^{T}\left(\left\|(-\Delta)^{\frac{\alpha_{1}}{2}} u_{t}\right\|_{2}^{2}+\left\|(-\Delta)^{\frac{\alpha_{2}}{2}} v_{t}\right\|_{2}^{2}\right) d t+\int_{0}^{T} \int_{\Omega}\left(G_{1}\left(u_{t}\right) u_{t}+G_{2}\left(v_{t}\right) v_{t}\right) d x d t \\
\leq E(T)+E(0)+\int_{0}^{T} E(t) d t+T C_{B} \sup _{\sigma \in[0, T]}\left(\|u(\sigma)\|_{2 \theta}^{2}+\|v(\sigma)\|_{2 \theta}^{2}\right) .
\end{gathered}
$$


Substituting the above estimate in (3.36), we find that

$$
\int_{0}^{T} E(t) d t \leq C_{B}(E(T)+E(0))+C_{B, T} \sup _{\sigma \in[0, T]}\left(\|u(\sigma)\|_{2 \theta}^{2}+\|v(\sigma)\|_{2 \theta}^{2}\right) .
$$

This estimate and (3.42) yields

$$
T E(T) \leq C_{B}(E(T)+E(0))+C_{B, T} \sup _{\sigma \in[0, T]}\left(\|u(\sigma)\|_{2 \theta}^{2}+\|v(\sigma)\|_{2 \theta}^{2}\right) .
$$

Choosing $T>2 C_{B}$, we find that

$$
E(T) \leq \gamma_{T} E(0)+C_{B, T} \sup _{\sigma \in[0, T]}\left(\|u(\sigma)\|_{2 \theta}^{2}+\|v(\sigma)\|_{2 \theta}^{2}\right),
$$

where

$$
\gamma_{T}=\frac{C_{B}}{T-C_{B}}<1
$$

Defining

$$
\chi_{m}=\sup _{\sigma \in[m,(m+1) T]}\left(\|u(\sigma)\|_{2 \theta}^{2}+\|v(\sigma)\|_{2 \theta}^{2}\right), \quad m=0,1,2, \ldots,
$$

the estimate (3.44) we can rewrite as

$$
E(T) \leq \gamma_{T} E(0)+C_{B, T} \chi_{0} .
$$

By iterating the above estimate on intervals $[m T,(m+1) T], m \in \mathbb{N}$, we deduce

$$
\begin{aligned}
E(m T) & \leq \gamma_{T}^{m} E(0)+C_{B, T} \sum_{k=1}^{m} \gamma_{T}^{m+1-l} \chi_{k-1} \\
& \leq \gamma_{T}^{m} E(0)+\frac{C_{B, T}}{1-\gamma_{T}} \sup _{\sigma \in[0, m T]}\left(\|u(\sigma)\|_{2 \theta}^{2}+\|v(\sigma)\|_{2 \theta}^{2}\right) .
\end{aligned}
$$

For any $t \geq 0$, there exists $m \in \mathbb{N}$ and $r \in[0, T)$ such that $t=m T+r$. Then, by (2.22) we obtain

$$
E(t) \leq E(m T) \leq \gamma_{T}^{-1} \gamma_{T}^{\frac{t}{T}} E(0)+\frac{C_{B, T}}{1-\gamma_{T}} \sup _{\sigma \in[0, t]}\left(\|u(\sigma)\|_{2 \theta}^{2}+\|v(\sigma)\|_{2 \theta}^{2}\right) .
$$

Therefore,

$$
E(t) \leq \vartheta_{B} E(0) e^{-\omega_{B} t}+C_{B} \sup _{\sigma \in[0, t]}\left(\|u(\sigma)\|_{2 \theta}^{2}+\|v(\sigma)\|_{2 \theta}^{2}\right), \quad \forall t \geq 0,
$$

with

$$
\vartheta_{B}=\gamma_{T}^{-1}, \quad \omega_{B}=-\frac{\ln \left(\gamma_{T}\right)}{T}, \quad C_{B}=\frac{C_{B, T}}{1-\gamma_{T}} .
$$

The proof is complete.

\subsection{Gradient system and stationary solutions}

We recall that a dynamical system $(H, S(t))$ is gradient if it possesses a strict Lyapunov functional. That is, a functional $\Phi: H \rightarrow \mathbb{R}$ is a strict Lyapunov function for a system $(H, S(t))$ if,

(i) the map $t \rightarrow \Phi(S(t) z)$ is non-increasing for each $z \in H$,

(ii) if $\Phi(S(t) z)=\Phi(z)$ for some $z \in H$ and for all $t$, then $z$ is a stationary point of $S(t)$, that is, $S(t) z=z$.

Lemma 3.3. Suppose that Assumption 2.1 holds. Then the dynamical system $\left(\mathcal{H}, S_{\epsilon}(t)\right)$ is gradient, that is, there exists a strict Lyapunov function $\Phi_{\epsilon}$ defined in $\mathcal{H}$. In addition,

$$
\Phi_{\epsilon}(U) \rightarrow \infty \Longleftrightarrow\|U\|_{\mathcal{H}} \rightarrow \infty .
$$


Proof. In order to prove this lemma we will show that the functional total energy $\mathcal{E}_{\epsilon}(t)$ defined in (2.15) is a Lyapunov function $\Phi_{\epsilon}$. Indeed, let $U_{0}=\left(u_{0}, v_{0}, u_{1}, v_{1}\right) \in \mathcal{H}$, then (2.16) shows that $t \mapsto \Phi_{\epsilon}\left(S(t) U_{0}\right)$ is a non-increasing function.

Now suppose that $\Phi_{\epsilon}\left(S(t) U_{0}\right)=\Phi_{\epsilon}\left(U_{0}\right), \forall t \geq 0$. Using again (2.16), we conclude that

$$
\left\|(-\Delta)^{\frac{\alpha_{1}}{2}} u_{t}\right\|_{2}^{2}+\left\|(-\Delta)^{\frac{\alpha_{2}}{2}} v_{t}\right\|_{2}^{2}=0, \quad \forall t \geq 0 .
$$

Consequently,

$$
u_{t}=v_{t}=0, \quad \text { for all } t \geq 0 \text {, a.e. in } \Omega .
$$

Therefore $u_{t}(t)=u_{0}$ and $v_{t}(t)=v_{0}$ for all $t \geq 0$. Then we can obtain that $S_{\epsilon}(t) U_{0}=U(t)=\left(u_{0}, v_{0}, 0,0\right)$ is a stationary solution, i.e., $S_{\epsilon}(t) U_{0}=U_{0}$, for all $t \geq 0$.

Now, from the second inequality in (2.17) we have

$$
\Phi_{\epsilon}(U) \leq C_{F}\left(1+\|U\|_{\mathcal{H}}^{p+1}\right), \quad \forall t \geq 0 .
$$

Considering the last estimate and taking $\Phi_{\epsilon}(U) \rightarrow \infty$ we have $\|U\|_{\mathcal{H}} \rightarrow \infty$. On the other hand, by the first inequality 2.17 we get

$$
\|U\|_{\mathcal{H}}^{2} \leq \frac{\Phi_{\epsilon}(U)+C_{F}}{C_{0}},
$$

from where we conclude that $\|U\|_{\mathcal{H}} \rightarrow \infty$ implies $\Phi_{\epsilon}(U) \rightarrow \infty$, proving (3.46).

Lemma 3.4. Suppose that Assumption 2.1 holds. Then the set $\mathcal{N}_{\epsilon}$ of the stationary points of $\left(\mathcal{H}, S_{\epsilon}(t)\right)$ is bounded in $\mathcal{H}$.

Proof. Let $U \in \mathcal{N}_{\epsilon}$ be arbitrary. We know that $U=(u, v, 0,0)$ and $U$ satisfies the equations

$$
\begin{aligned}
& -\Delta u=f_{1}(u, v)+\epsilon h_{1}, \\
& -\Delta v=f_{2}(u, v)+\epsilon h_{2} .
\end{aligned}
$$

Multiplying (3.49) and (3.50) by $u$ and $v$, respectively, and then integrating over $\Omega$, we get

$$
\|\nabla u\|^{2}+\|\nabla v\|^{2}=\int_{\Omega}\left(f_{1}(u, v) u+f_{2}(u, v) v\right) d x+\epsilon \int_{\Omega}\left(h_{1} u+h_{2} v\right) d x,
$$

By (2.8) and (2.9) we have

$$
\begin{aligned}
\int_{\Omega}\left(f_{1}(u, v) u+f_{2}(u, v) v\right) d x & \leq 2 \beta_{0}\left(\|u\|_{2}^{2}+\|v\|_{2}^{2}\right)+2 m_{F}|\Omega| \\
& \leq \frac{2 \beta_{0}}{\lambda_{1}}\left(\|\nabla u\|_{2}^{2}+\|\nabla v\|_{2}^{2}\right)+2 m_{F}|\Omega|,
\end{aligned}
$$

and therefore, in light of (2.18),

$$
4 C_{0}\|U\|_{\mathcal{H}}^{2} \leq 2 m_{F}|\Omega|+\epsilon \int_{\Omega}\left(h_{1} u+h_{2} v\right) d x .
$$

Hence, using the estimate (2.19), we deduce

$$
3 C_{0}\|U\|_{\mathcal{H}}^{2} \leq 2 m_{F}|\Omega|+\frac{1}{4 C_{0} \lambda_{1}}\left(\left\|h_{1}\right\|_{2}^{2}+\left\|h_{2}\right\|_{2}^{2}\right) .
$$

The proof is complete. 


\subsection{Proof of Theorem 3.1}

(i) We consider a bounded positively invariant set $B \subset \mathcal{H}$ with respect to $S_{\epsilon}(t)$, denote $S_{\epsilon}(t) U^{i}=$ $\left(u^{i}(t), v^{i}(t), u_{t}^{i}(t), v_{t}^{i}(t)\right)$ for $U^{i} \in B, i=1,2$, and set $u=u^{1}-u^{2}, v=v^{1}-v^{2}$, as before. It follows from (2.22) that

$$
\left\|S_{\epsilon}(t) U^{1}-S_{\epsilon}(t) U^{2}\right\|_{\mathcal{H}}^{2} \leq a(t)\left\|U^{1}-U^{2}\right\|_{\mathcal{H}}^{2}
$$

with $a(t)=e^{C_{0} T}$. We denote by $X=H_{0}^{1}(\Omega) \times H_{0}^{1}(\Omega)$ and define the semi-norm

$$
n_{X}(u, v):=\left(\|u\|_{2 \theta}^{2}+\|v\|_{2 \theta}^{2}\right)^{\frac{1}{2}} .
$$

Since the embedding (in 2D) $H_{0}^{1}(\Omega) \hookrightarrow L^{2 \theta}(\Omega)$ is compact, we know that $n_{X}$ is a compact semi-norm on $X$.

By Lemma 3.2, we can get

$$
\left\|S_{\epsilon}(t) U^{1}-S_{\epsilon}(t) U^{2}\right\|_{\mathcal{H}}^{2} \leq b(t)\left\|U_{1}-U_{2}\right\|_{\mathcal{H}}^{2}+c(t) \sup _{s \in[0, t]}\left[n_{X}(u(s), v(s))\right]^{2},
$$

where $b(t)=\vartheta_{B} e^{-\omega_{B} t}$ and $c(t)=C_{B}$. It is easy to get that

$$
b(t) \in L^{1}\left(\mathbb{R}^{+}\right) \text {and } \lim _{t \rightarrow \infty} b(t)=0 .
$$

Since $B \subset \mathcal{H}$ is bounded, we know that $c(t)$ is locally bounded on [0, $\infty$. From [21, Definition 7.9.2], we get that the dynamics system $\left(\mathcal{H}, S_{\epsilon}(t)\right)$ is quasi-stable on any bounded positively invariant set $B \subset \mathcal{H}$.

(ii) Since the system $\left(\mathcal{H}, S_{\epsilon}(t)\right)$ is quasi-stable, applying Proposition 7.9.4 in [21], then ( $\left.\mathcal{H}, S_{\epsilon}(t)\right)$ is asymptotically smooth. Thus, noting Lemmas 3.3 and 3.4 and using Corollary 7.5.7 in [21], we know that $\left(\mathcal{H}, S_{\epsilon}(t)\right)$ has a compact global attractor given by $\mathcal{A}_{\epsilon}=\mathbb{M}_{+}\left(\mathcal{N}_{\epsilon}\right)$.

(iii) Combining Theorem 3.1-(ii) and Theorem 7.5.10 in [21], we can get the result.

(iv) From the above, $\left(\mathcal{H}, S_{\epsilon}(t)\right)$ is quasi-stable on the attractor $\mathcal{A}_{\epsilon}$. Thus, using Theorem 7.9.6 in [21], we know that the attractor $\mathcal{A}_{\epsilon}$ has finite fractal dimension $\operatorname{dim}_{\mathcal{H}}^{f} \mathcal{A}_{\epsilon}$.

(v) Since the system $\left(\mathcal{H}, S_{\epsilon}(t)\right)$ is quasi-stable on the attractor $\mathcal{A}_{\epsilon}$, it follows from Theorem 7.9.8 in [21] that any complete trajectory $U=\left(u, v, u_{t}, v_{t}\right)$ in $\mathcal{A}_{\epsilon}$ enjoys the following regularity properties

$$
u_{t} \in L^{\infty}\left(\mathbb{R}, H_{0}^{1}(\Omega)\right) \cap C\left(\mathbb{R}, L^{2}(\Omega)\right), \quad v_{t} \in L^{\infty}\left(\mathbb{R}, H_{0}^{1}(\Omega)\right) \cap C\left(\mathbb{R}, L^{2}(\Omega)\right),
$$

and

$$
u_{t t} \in L^{\infty}\left(\mathbb{R}, L^{2}(\Omega)\right), \quad v_{t t} \in L^{\infty}\left(\mathbb{R}, L^{2}(\Omega)\right) .
$$

Moreover, there exists $R>0$ such that

$$
\left\|\left(u_{t}, v_{t}\right)\right\|_{\left(H_{0}^{1}(\Omega)\right)^{2}}^{2}+\left\|\left(u_{t t}, v_{t t}\right)\right\|_{\left(L^{2}(\Omega)\right)^{2}}^{2} \leq R^{2} .
$$

From (3.54), (1.1), the embedding $L^{\infty}\left(\mathbb{R} ; H_{0}^{1}(\Omega)\right) \hookrightarrow L^{\infty}\left(\mathbb{R} ; D\left((-\Delta)^{\alpha_{i}}\right)\right)$ for $0<\alpha_{i}<1 / 2$ and the fact that $0<\epsilon<$ 1 , we obtain

$$
\begin{aligned}
& \|\Delta u\|_{2} \leq\left\|u_{t t}\right\|_{2}+\left\|(-\Delta)^{\alpha_{1}} u_{t}\right\|+\left\|g_{1}\left(u_{t}\right)\right\|_{2}+\left\|f_{1}(u, v)\right\|_{2}+\left\|h_{1}\right\|_{2} \leq C, \\
& \|\Delta v\|_{2} \leq\left\|v_{t t}\right\|_{2}+\left\|(-\Delta)^{\alpha_{2}} v_{t}\right\|+\left\|g_{2}\left(v_{t}\right)\right\|_{2}+\left\|f_{2}(u, v)\right\|_{2}+\left\|h_{2}\right\|_{2} \leq C .
\end{aligned}
$$

Therefore (3.24) is obtained by (3.54) and (3.55). Since the global attractors $\mathcal{A}_{\epsilon}$ are also characterized as

$$
\mathcal{A}_{\epsilon}=\left\{U(0): U \text { is a bounded full trajectory of } S_{\epsilon}(t)\right\},
$$

we conclude the $\mathcal{A}_{\epsilon}$ is bounded in $\mathcal{H}_{1}$.

(vi) Now we take $\mathcal{B}=\left\{U: \Phi_{\epsilon}(U) \leq R\right\}$, where $\Phi_{\epsilon}$ is the strict Lyapunov functional considered in Lemma 3.3. Then we know that the set $\mathcal{B}$ is a positively invariant absorbing set for $R$ large enough. Hence the system $\left(\mathcal{H}, S_{\epsilon}(t)\right)$ is quasi-stable on $\mathcal{B}$. 
For solution $U(t)$ with initial data $z=U(0) \in \mathcal{B}$, we can conclude from the positive invariance of $\mathcal{B}$ that there exists $C_{\mathcal{B}}>0$ such that for any $0 \leq t \leq T$,

$$
\left\|U_{t}(t)\right\|_{\widetilde{\mathcal{H}}_{-1}} \leq C_{\mathcal{B}},
$$

which gives us for any $0 \leq t_{1}<t_{2} \leq T$,

$$
\left\|S_{\epsilon}\left(t_{1}\right) z-S_{\epsilon}\left(t_{2}\right) z\right\|_{\widetilde{\mathcal{H}}_{-1}} \leq \int_{t_{1}}^{t_{2}}\left\|U_{t}(\tau)\right\|_{\widetilde{\mathcal{H}}_{-1}} d \tau \leq C_{\mathcal{B}}\left|t_{1}-t_{2}\right| .
$$

From (3.56), we conclude that for any $z \in \mathcal{B}$, the map $t \mapsto S_{\epsilon}(t) z$ is Hölder continuous in the extended space $\widetilde{\mathcal{H}}_{-1}$ with exponent $\delta=1$. Then we can get the existence of a generalized exponential attractor whose fractal dimension is finite in $\widetilde{\mathcal{H}}_{-1}$.

Following the same arguments in [21], we can obtain the existence of exponential attractors in $\widetilde{\mathcal{H}}_{-\delta}$ with $\delta \in(0,1)$.

Thus, the proof of Theorem 3.1 is complete.

\section{Upper and lower semicontinuity of global attractors}

In this section, we study the continuity of the attractors $\mathcal{A}_{\epsilon}$ as $\epsilon \rightarrow \epsilon_{0}$. Firstly, we prove that this family of attractors converges upper and lower semicontinuously to the global compact attractor $\mathcal{A}_{\epsilon_{0}}$ of the limiting semi-flow $S_{\epsilon_{0}}(t)$ on a residual subset of [0,1], by using the abstract result in [31, Theorem 5.2], where the results were obtained as a extension of the previous results in [4]. Finally, the upper semicontinuity is proved for all $\epsilon_{0} \in[0,1]$.

Let $\Lambda$ be a complete metric space and $S_{\lambda}(t)$ a parametrised family of semigroups on $X$. Suppose that (L1) $S_{\lambda}(t)$ has a global attractor $\mathcal{A}_{\lambda}$ for every $\lambda \in \Lambda$,

(L2) There is a bounded subset $D$ of $X$ such that $\mathcal{A}_{\lambda} \subset D$ for every $\lambda \in \Lambda$,

(L3) For $t>0, S_{\lambda}(t) x$ is continuous in $\lambda$, uniformly for $x$ in bounded subsets of $X$.

Then $\mathcal{A}_{\lambda}$ is continuous on all $\Lambda \star$ where $\Lambda \star$ is a residual set dense in $\Lambda$ (see [31, Theorem 5.2]).

The following lemma will be used to obtain a uniform (with respect to the parameter $\epsilon$ of the problem) bound for the attractor that will be used to verify the property (L2) above (see [21, Remark 7.5.8]).

Lemma 4.1. Under the assumptions of Lemma 3.3, the following relation is valid:

$$
\sup _{U \in \mathcal{A}_{\epsilon}} \Phi_{\epsilon}(U) \leq \sup _{U \in \mathcal{N}_{\epsilon}} \Phi_{\epsilon}(U) .
$$

Theorem 4.2 (Residual continuity). Under the assumptions of Theorem 3.1, there exists a set $I_{\star}$ dense in $[0,1]$ such that $\mathcal{A}_{\epsilon}$ is continuous at $\epsilon_{0} \in I_{\star}$, that is,

$$
\lim _{\epsilon \rightarrow \epsilon_{0}} \mathrm{~d}_{\mathcal{H}}\left(\mathcal{A}_{\epsilon}, \mathcal{A}_{\epsilon_{0}}\right)=0, \quad \forall \epsilon_{0} \in I_{\star},
$$

where $\mathrm{d}_{\mathcal{H}}$ denotes the Hausdorff distance between bounded sets $B, C \subset \mathcal{H}$, defined as

$$
\mathrm{d}_{\mathcal{H}}(B, C)=\max \left\{\operatorname{dist}_{\mathcal{H}}(B, C), \operatorname{dist}_{\mathcal{H}}(C, B)\right\} .
$$

Proof. We shall apply the abstract result in [31, Theorem 5.2] with $\Lambda=[0,1]$. The argument follows the lines of the proof of Theorem 4.1 in [36]. Theorem 3.1-(ii) indicates that (L1) holds. Now, we obtain uniform (with 
respect to the parameter $\epsilon$ of the problem) bounds for the attractor. By (2.17) and Lemma 4.1, we obtain

$$
\begin{aligned}
\sup _{U \in \mathcal{A}_{\epsilon}}\|U\|_{\mathcal{H}}^{2} & \leq \frac{\sup _{U \in \mathcal{A}_{\epsilon}} \Phi_{\epsilon}(U)+C_{F}}{C_{0}} \\
& \leq \frac{\sup _{U \in \mathcal{N}_{\epsilon}} \Phi_{\epsilon}(U)+C_{F}}{C_{0}} \\
& \leq \frac{C_{F} \sup _{U \in \mathcal{N}_{\epsilon}}\|U\|_{\mathcal{H}}^{p+1}+2 C_{F}}{C_{0}} .
\end{aligned}
$$

Hence, by (3.51), we can conclude that there exists a constant $C_{0}>0$ independent of $\epsilon$ such that

$$
\sup _{U \in \mathcal{A}_{\epsilon}}\|U\|_{\mathcal{H}}^{2} \leq C_{0}, \quad \forall \epsilon \in[0,1] .
$$

Then, $D=\left\{U \in \mathcal{H}:\|U\|_{\mathcal{H}}^{2} \leq C_{0}\right\}$ is a bounded set independent of $\epsilon$ such that

$$
\mathcal{A}_{\epsilon} \subset D, \quad \forall \epsilon \in[0,1],
$$

and thereby (L2) holds.

Let $B$ be a bounded set of $\mathcal{H}$. Given $\epsilon_{1}, \epsilon_{2} \in[0,1]$ and $U_{0} \in B$, let us denote

$$
S_{\epsilon_{i}}(t) U_{0}=\left(u^{i}(t), v^{i}(t), u_{t}^{i}(t), v_{t}^{i}(t)\right), \quad i=1,2,
$$

and

$$
u=u^{1}-u^{2}, \quad v=v^{1}-v^{2} .
$$

Then $U=\left(u, v, u_{t}, v_{t}\right)$ satisfies the following system

$$
\left\{\begin{array}{l}
u_{t t}-\Delta u+(-\Delta)^{\alpha_{1}} u_{t}=F_{1}(u, v)-G_{1}\left(u_{t}\right)+\left(\epsilon_{1}-\epsilon_{2}\right) h_{1}, \\
v_{t t}-\Delta v+(-\Delta)^{\alpha_{2}} v_{t}=F_{2}(u, v)-G_{2}\left(v_{t}\right)+\left(\epsilon_{1}-\epsilon_{2}\right) h_{2},
\end{array}\right.
$$

where

$$
F_{i}(u, v)=f_{i}\left(u^{1}, v^{1}\right)-f_{i}\left(u^{2}, v^{2}\right), \quad i=1,2,
$$

and

$$
G_{1}\left(u_{t}\right)=g_{1}\left(u_{t}^{1}\right)-g_{1}\left(u_{t}^{2}\right), G_{2}\left(v_{t}\right)=g_{2}\left(v_{t}^{1}\right)-g_{2}\left(v_{t}^{2}\right) .
$$

Multiplying the first equation in (4.58) by $u_{t}$, the second by $v_{t}$, respectively, and using integration by parts, we obtain

$$
\begin{aligned}
\frac{1}{2} \frac{\mathrm{d}}{\mathrm{d} t}\|U\|_{\mathcal{H}}^{2}= & \int_{\Omega}\left(F_{1}(u, v) u_{t}+F_{2}(u, v) v_{t}\right) \mathrm{d} x \\
& -\left(\left\|(-\Delta)^{\frac{\alpha_{1}}{2}} u_{t}\right\|_{2}^{2}+\left\|(-\Delta)^{\frac{\alpha_{2}}{2}} v_{t}\right\|_{2}^{2}\right) \\
& -\int_{\Omega}\left(G_{1}\left(u_{t}\right) u_{t}+G_{2}\left(v_{t}\right) v_{t}\right) \mathrm{d} x \\
& -\left(\epsilon_{1}-\epsilon_{2}\right) \int_{\Omega}\left(h_{1} u_{t}+h_{2} v_{t}\right) \mathrm{d} x .
\end{aligned}
$$

Using (2.7), Hölder’s inequality and the embedding (in 2D) $H_{0}^{1}(\Omega) \hookrightarrow L^{s}(\Omega)$ for $1 \leq s<\infty$, we deduce

$$
\begin{aligned}
\int_{\Omega} F_{1}(u, v) u_{t} \mathrm{~d} x & \leq C\left(1+\left\|S_{\epsilon_{1}}(t) U_{0}\right\|_{\mathcal{H}}^{p-1}+\left\|S_{\epsilon_{2}}(t) U_{0}\right\|_{\mathcal{H}}^{p-1}\right)\left(\|u\|_{2 p}+\|v\|_{2 p}\right)\left\|u_{t}\right\|_{2} \\
& \leq C\left(1+\left\|S_{\epsilon_{1}}(t) U_{0}\right\|_{\mathcal{H}}^{p-1}+\left\|S_{\epsilon_{2}}(t) U_{0}\right\|_{\mathcal{H}}^{p-1}\right)\left(\|\nabla u\|_{2}+\|\nabla v\|_{2}\right)\left\|u_{t}\right\|_{2} .
\end{aligned}
$$

Using the fact that $\mathcal{E}_{\epsilon}(t)$ is a non-increasing function and (2.17), we find that for $i=1$, 2,

$$
\left\|S_{\epsilon_{i}}(t) U_{0}\right\|_{\mathcal{H}}^{p-1} \leq \frac{\mathcal{E}_{\epsilon_{i}}(0)+C_{F}}{C_{0}} \leq \frac{C_{F}\left(1+\left\|U_{0}\right\|_{\mathcal{H}}^{p+1}\right)+C_{F}}{C_{0}} \leq C_{B}, \quad \forall U_{0} \in B
$$


Inserting the above estimate into (4.60) and using Young's inequality, we see that

$$
\begin{aligned}
\int_{\Omega} F_{1}(u, v) u_{t} \mathrm{~d} x & \leq C_{B}\left(\|\nabla u\|_{2}+\|\nabla v\|_{2}\right)\left\|u_{t}\right\|_{2} \\
& \leq C_{B}\left(\|\nabla u\|_{2}^{2}+\|\nabla v\|_{2}^{2}\right)+\left\|u_{t}\right\|_{2}^{2} .
\end{aligned}
$$

Analogously,

$$
\int_{\Omega} F_{2}(u, v) v_{t} \mathrm{~d} x \leq C_{B}\left(\|\nabla u\|_{2}^{2}+\|\nabla v\|_{2}^{2}\right)+\left\|v_{t}\right\|_{2}^{2} .
$$

Adding the last two estimates, we conclude that

$$
\int_{\Omega}\left(F_{1}(u, v) u_{t}+F_{2}(u, v) v_{t}\right) \mathrm{d} x \leq C_{D}\|U\|_{\mathcal{H}}^{2} .
$$

By the monotonicity property (2.13), we get

$$
-\int_{\Omega}\left(G_{1}\left(u_{t}\right) u_{t}+G_{2}\left(v_{t}\right) v_{t}+G_{3}\left(w_{t}\right) w_{t}\right) \mathrm{d} x \leq 0 .
$$

In addition,

$$
\begin{aligned}
\left(\epsilon_{1}-\epsilon_{2}\right) \int_{\Omega}\left(h_{1} u_{t}+h_{2} v_{t}\right) \mathrm{d} x & \leq \frac{1}{2}\left(\left\|u_{t}\right\|_{2}^{2}+\left\|v_{t}\right\|_{2}^{2}\right)+\frac{1}{2}\left|\epsilon_{1}-\epsilon_{2}\right|^{2}\left(\left\|h_{1}\right\|_{2}^{2}+\left\|h_{2}\right\|_{2}^{2}\right) \\
& \leq \frac{1}{2}\|U\|_{\mathcal{H}}^{2}+\frac{1}{2}\left|\epsilon_{1}-\epsilon_{2}\right|^{2}\left(\left\|h_{1}\right\|_{2}^{2}+\left\|h_{2}\right\|_{2}^{2}\right) .
\end{aligned}
$$

Substituting the estimates (4.61)-(4.63) into (4.59), we obtain

$$
\frac{\mathrm{d}}{\mathrm{d} t}\|U\|_{\mathcal{H}}^{2} \leq C_{B}\|U\|_{\mathcal{H}}^{2}+\left|\epsilon_{1}-\epsilon_{2}\right|^{2}\left(\left\|h_{1}\right\|_{2}^{2}+\left\|h_{2}\right\|_{2}^{2}\right) .
$$

Applying Gronwall's inequality to (4.64) and using that $\|U(0)\|_{\mathcal{H}}^{2}=0$, we conclude that

$$
\|U(t)\|_{\mathcal{H}}^{2} \leq \frac{1}{C_{B}}\left(e^{C_{B} t}-1\right)\left(\left\|h_{1}\right\|_{2}^{2}+\left\|h_{2}\right\|_{2}^{2}\right)\left|\epsilon_{1}-\epsilon_{2}\right|^{2}, \quad t>0 .
$$

This implies

$$
\left\|S_{\epsilon_{1}}(t) U_{0}-S_{\epsilon_{2}}(t) U_{0}\right\|_{\mathcal{H}} \leq \sqrt{\frac{1}{C_{D}}\left(e^{C_{D} t}-1\right)\left(\left\|h_{1}\right\|_{2}^{2}+\left\|h_{2}\right\|_{2}^{2}\right)}\left|\epsilon_{1}-\epsilon_{2}\right|, \quad t>0,
$$

and thereby (L3) holds. Therefore all the assumptions of Theorem [31, Theorem 5.2] are fulfilled. Then there exists a dense set $I \star \subset[0,1]$ such that (4.57) holds. The proof is complete.

Theorem 4.3 (Upper-semicontinuity). Under the assumptions of Theorem 3.1, the family of global attractors $\mathcal{A}_{\epsilon}$ is upper semicontinuous at $\epsilon \in[0,1]$, that is,

$$
\lim _{\epsilon \rightarrow \epsilon_{0}} \operatorname{dist}_{\mathcal{H}}\left(\mathcal{A}_{\epsilon}, \mathcal{A}_{\epsilon_{0}}\right)=0, \quad \forall \epsilon \in[0,1] .
$$

Proof. The argument is inspired by (see, e.g. [25, 30]). We proceed by contradiction as in [35]. Suppose that (4.65) does not hold. Then there exist $\delta>0$ and a sequences $\epsilon_{n} \rightarrow \epsilon_{0}$ and $U_{0}^{n} \in \mathcal{A}_{\epsilon_{n}}$ such that

$$
\operatorname{dist}_{\mathcal{H}}\left(U_{0}^{n}, \mathcal{A}_{\epsilon_{0}}\right) \geq \delta>0, \quad \forall n .
$$

Let $U^{n}(t)=\left(u^{n}(t), v^{n}(t), u_{t}^{n}(t), v_{t}^{n}(t)\right)$ be a full trajectory from the attractor $\mathcal{A}_{\epsilon_{n}}$ such that $U^{n}(0)=U_{0}^{n}$. From uniform estimate (3.24), we know 
Since $\mathcal{H}_{1}$ is compactly embedded into $\mathcal{H}$, using Simon's Compactness Theorem (see [37]), we obtain a subsequence $\left\{U^{n_{k}}\right\}$ and $U \in C([-T, T] ; \mathcal{H})$ such that

$$
\lim _{k \rightarrow \infty} \max _{t \in[-T, T]}\left\|U^{n_{k}}(t)-U(t)\right\|_{\mathcal{H}}=0 .
$$

By (4.67) and (4.68), we conclude that

$$
\sup _{t \in \mathbb{R}}\|U(t)\|_{\mathcal{H}}<\infty .
$$

Using the same argument as in the proof of property (L3) above, we can see that

$$
U(t)=\left(u(t), v(t), u_{t}(t), v_{t}(t)\right)
$$

solves the limiting equations $\left(\epsilon=\epsilon_{0}\right)$

$$
\left\{\begin{array}{l}
u_{t t}-\Delta u+(-\Delta)^{\alpha_{1}} u_{t}+g_{1}\left(u_{t}\right)=f_{1}(u, v)+\epsilon_{0} h_{1}, \\
v_{t t}-\Delta v+(-\Delta)^{\alpha_{2}} v_{t}+g_{2}\left(v_{t}\right)=f_{2}(u, v)+\epsilon_{0} h_{2} .
\end{array}\right.
$$

Therefore $U(t)$ is a bounded full trajectory for the limiting semi-flow $S_{\epsilon_{0}}(t)$. Consequently,

$$
U_{0}^{n_{k}} \rightarrow U(0) \in \mathcal{A}_{0},
$$

which is contradict (4.66). The proof is complete.

Conflict of interest: On behalf of all authors, the corresponding author states that there is no conflict of interest.

Acknowledgments: A.J.A. Ramos thanks the CNPq for financial support through the projects "Asymptotic stabilization and numerical treatment for carbon nanotubes” (CNPq Grant 310729/2019-0).

Data Availability Statement: Data sharing is not applicable to this article as no datasets were generated or analysed during the current study.

\section{References}

[1] K. Agre and M. A. Rammaha, Systems of nonlinear wave equations with damping and source terms, Differential Integral Equations, 19 (2006), pp. 1235-1270.

[2] C. O. Alves, M. M. Cavalcanti, V. N. Domingos Cavalcanti, M. A. Rammaha, and D. Toundykov, On existence, uniform decay rates and blow up for solutions of systems of nonlinear wave equations with damping and source terms, Discrete Contin. Dyn. Syst. Ser. S, 2 (2009), pp. 583-608.

[3] F. Araruna and F. D. Bezerra, Rate of attraction for a semilinear wave equation with variable coefficients and critical nonlinearities, Pacific Journal of Mathematics, 266 (2013), pp. 257-282.

[4] A. V. Babin and S. Y. Pilyugin, Continuous dependence of attractors on the shape of domain, J. Math. Sci., 87 (1997), pp. 33043310 .

[5] A. V. Babin and M. I. Vishik, Attractors of evolution equations, North Holland, Amsterdam, 1992.

[6] V. Barbu, Nonlinear Differential Equations of Monotone Types in Banach Spaces, vol. 190 of Springer Monographs in Mathematics, Springer, New York, 2010.

[7] F. D. Bezerra, A. N. Carvalho, T. Dlotko, and M. J. Nascimento, Fractional schrödinger equation; solvability and connection with classical schrödinger equation, Journal of Mathematical Analysis and Applications, 457 (2018), pp. 336 - 360.

[8] J. E. Billotti and J. P. LaSalle, Dissipative periodic processes, Bull Am Math Soc, 77 (1971), pp. 1082-1088.

[9] L. Bociu, Local and global wellposedness of weak solutions for the wave equation with nonlinear boundary and interior sources of supercritical exponents and damping, Nonlinear Analysis, 71 (2009), pp. 560-575.

[10] L. Bociu and I. Lasiecka, Blow-up of weak solutions for the semilinear wave equations with nonlinear boundary and interior sources and damping, Appl. Math., 35 (2008), pp. 281-304.

[11] - Uniqueness of weak solutions for the semilinear wave equations with supercritical boundary/interior sources and damping, Discrete Contin. Dyn. Syst., 22 (2008), pp. 835-860. 
[12] - Local Hadamard well-posedness for nonlinear wave equations with supercritical sources and damping, J. Differential Equations, 249 (2010), pp. 654-683.

[13] A. Carvalho and J. Cholewa, Regularity of solutions on the global attractor for a semilinear damped wave equation, Journal of Mathematical Analysis and Applications, 337 (2008), pp. 932 - 948.

[14] A. Carvalho, J. Cholewa, and T. Dlotko, Strongly damped wave problems: Bootstrapping and regularity of solutions, Journal of Differential Equations, 244 (2008), pp. 2310 - 2333.

[15] A. N. Carvalho and J. W. Cholewa, Attractors for strongly damped wave equations with critical nonlinearities, Pacific Journal of Mathematics, 207 (2002), pp. 287-310.

[16] A. N. Carvalho and J. W. Cholewa, Local well posedness for strongly damped wave equations with critical nonlinearities, Bulletin of the Australian Mathematical Society, 66 (2002), pp. 443-463.

[17] S. Chen and R. Triggiani, Characterization of domains of fractional powers of certain operators arising in elastic systems, and applications, Journal of Differential Equations, 88 (1990), pp. $279-293$.

[18] S. Chen and R. Triggiani, Gevrey class semigroups arising from elastic systems with gentle dissipation: The case, Proceedings of the American Mathematical Society, (1990), pp. 401-415.

[19] S. P. Chen and R. Triggiani, Proof of extensions of two conjectures on structural damping for elastic systems., Pacific J. Math., 136 (1989), pp. 15-55.

[20] I. Chueshov and I. Lasiecka, Long-Time Behavior of Second Order Evolution Equations with Nonlinear Damping, vol. 195, Mem. Amer. Math. Soc., Providence, 2008.

[21] _ Von Karman Evolution Equations. Well-posedness and Long Time Dynamics, Dynamics, Springer Monographs in Mathematics, Springer, New York, 2010.

[22] M. Conti, S. Gatti, and V. Pata, Uniform decay properties of linear volterra integro-differential equations, Methods Appl. Sci., 18 (2008), pp. 21-45.

[23] M. M. Freitas, M. L. Santos, and J. A. Langa, Porous elastic system with nonlinear damping and sources terms, J. Differential Equations, 264 (2018), pp. 2970-3051.

[24] V. Georgiev and G. Todorova, Existence of a solution of the wave equation with nonlinear damping and source terms, J. Differential Equation, 109 (1994), pp. 295-308.

[25] P. G. Geredeli and I. Lasiecka, Asymptotic analysis and upper semicontinuity with respect to rotational inertia of attractors to von karman plates with geometrically localized dissipation and critical nonlinearity, Nonlinear Anal., 91 (2013), pp. 72-92.

[26] Y. Guo and M. A. Rammaha, Blow-up of solutions to systems of nonlinear wave equations with supercritical sources, Appl. Anal., 92, (2013), pp. 1101-1115.

[27] - Global existence and decay of energy to systems of wave equations with damping and supercritical sources, $Z$. Angew. Math. Phys., 64 (2013), pp. 621-658.

[28] - Systems of nonlinear wave equations with damping and supercritical sources, Transactions of the American Mathematical Society, 366 (2014), pp. 2265-2325.

[29] J. K. Hale, Asymptotic behavior of dissipative systems, Mathematical surveys and monographs, American Mathematival Society, Providence, RI, 1988.

[30] J. K. Hale and G. Raugel, Upper semicontinuity of the attractor for a singulary perturbed hyperbolic equation, J. Differential Equations, 73 (1988), pp. 197-214.

[31] L. Hoang, E. Olson, and J. Robinson, On the continuity of global attractors, Proceedings of the American Mathematical Society, 143 (2015), pp. 4389-4395.

[32] H. Koch and I. Lasiecka, Hadamard well-posedness of weak solutions in nonlinear dynamic elasticity-full von Karman systems, vol. 50, Evolution equations, semigroups and functional analysis (Milano, 2000), Progr. Nonlinear Differential Equations Appl., Birkhäuser, Basel, pp. 197-216, 2002.

[33] O. A. Ladyzhenskaya, The Mathematical Theory of Viscous Incompressible Flow, Gordon and Breach, Science Publishers, New York, 1963.

[34] I. Lasiecka and R. Triggiani, Exact null controllability of structurally damped and thermo-elastic parabolic models, Atti della Accademia Nazionale dei Lincei. Classe di Scienze Fisiche, Matematiche e Naturali. Rendiconti Lincei. Matematica e Applicazioni, 9 (1998), pp. 43-69.

[35] T. F. Ma and R. N. Monteiro, Singular limit and long-time dynamics of bresse systems, SIAM Journal on Mathematical Analysis, 49 (2017), pp. 2468-2495.

[36] T. F. Ma and P. N. Seminario-Huertas, Attractors for semilinear wave equations with localized damping and external forces, Communications on Pure and Applied Analysis, 19 (2020), pp. 2219-2233.

[37] J. Málek and J. Nečas, Compact sets in the space $L^{p}$ (0, T, B), Annali di Matematica Pura ed Applicata, 146 (1987), pp. 65-96.

[38] R. Michael, Structurally damped elastic waves in 2d, Mathematical Methods in the Applied Sciences, 39, pp. 4618-4628.

[39] D. R. Pitts and M. A. Rammaha, Global existence and non-existence theorems for nonlinear wave equations, Indiana Univ. Math. J., 51 (2002), pp. 1479-1509.

[40] M. A. Rammaha and S. Sakuntasathien, Critically and degenerately damped systems of nonlinear wave equations with source terms, Appl. Anal., 89 (2010), pp. 1201-1227.

[41] Global existence and blow up of solutions to systems of nonlinear wave equations with degenerate damping and source terms, Nonlinear Anal., 72 (2010), pp. 2658-2683. 
[42] M. A. Rammaha and T. A. Strei, Global existence and nonexistence for nonlinear wave equations with damping and source terms, Trans. Am. Math. Soc., 354 (2002), pp. 3621-3637.

[43] M. A. Rammaha and Z. Welstein, Hadamard well-posedness for wave equations with p-laplacian damping and supercritical sources, Adv. Differ. Equ., 17 (2012), pp. 105-150.

[44] J. C. Robinson, Infinite-dimensional dynamical systems: an introduction to dissipative parabolic PDEs and the theory of global attractors, vol. 28, Cambridge University Press, 2001.

[45] R. Temam, Infinite-dimensional dynamical systems in mechanics and physics, SIAM, Philadelphia, PA, 1995.

[46] R. Triggiani, Regularity of some structurally damped problems with point control and with boundary control, Journal of Mathematical Analysis and Applications, 161 (1991), pp. 299 - 331. 